\title{
Lentes intraoculares fácicas para miopia e astigmatismo: revisão prospectiva
}

Phakic intraocular lenses for myopia and astigmatism: a prospective review

Lorena Barros ${ }^{1}$ (D), Nelson Sena Júnior ${ }^{1}$ (D), Cesar Motta ${ }^{1}$ (D), Guilherme Criado ${ }^{1}$, Renato Ambrósio Júnior ${ }^{2}$ (1)

Descritores: Lentes intraoculares; Miopia/ cirurgia; Astgmatismo/cirurgia;

Cirurgia refrativa

Keywords:

Intraocular lenses; Myopia/ surgery; Astigmatism/surgery;

Refractive surgery Submetido:
03/09/2020

Aceito: 26/4/2021

Autor correspondente: Renato Ambrósio Júnior Instituto de Olhos Renato Ambrósio Rua Conde de Bonfim, 211/712 - Tijuca CEP: 20520-050 - Rio de Janeiro, RJ, Brasil E-mail: dr.renatoambrosio@gmail.com Instituição:
Hospital Universitario Gafrée e Guinle, Rio de Janeiro, RJ, Brasil.

Fonte de auxílio à pesquisa: não financiado.

Conflitos de interesse: os autores declaram que não há conflitos de interesses.

\section{RESUMO}

O implante de lentes intraoculares fácicas com finalidade refrativa é uma alternativa cirúrgica eletiva segura e eficiente. Essa opção deve ser considerada de forma eletiva para tratar ametropias, nos casos em que as cirurgias de correção visual refrativa a laser na córnea apresentam maior risco de complicações, seja por características da córnea ou pela magnitude da ametropia. Este artigo traz uma revisão prospectiva das lentes fácicas disponíveis no mercado brasileiro em 2020 e suas características.

\section{ABSTRACT}

Implantation of phakic intraocular lenses for refractive purpose is a safe and efficient elective surgical alternative. This option should be considered to electively treat ametropia when laser corneal refractive surgery has higher risk of complications, either due to corneal characteristics or the magnitude of ametropia. This article provides a prospective review of the phakic lenses available in the Brazilian market in 2020 and their characteristics. 


\section{INTRODUÇÃO}

A cirurgia refrativa é uma subespecialidade da oftalmologia que trata dos procedimentos eletivos cujo propósito é reduzir a dependência de correção por óculos ou lentes de contato. O objetivo mais importante é aliar satisfação com qualidade de visão para o paciente. ${ }^{(1)}$ As opções de cirurgias de correção visual a laser (CVL) refrativa na córnea são laser assisted in situ keratomileusis (Lasik), photorefractive keratectomy (PRK) e small incision lenticular extraction (SMILE). Essas cirurgias apresentam limites e restrições para a correção de altas ametropias, considerando-se a qualidade visual. ${ }^{(2)}$ No caso de altas ametropias, observa-se redução da capacidade funcional do paciente, podendo gerar dificuldades ao exercer tarefas simples do cotidiano.

A estabilidade da córnea após os procedimentos de CVL deve ser considerada de acordo com sua suscetibilidade para desenvolver descompensação biomecânica e ectasia iatrogênica e o enfraquecimento gerado pela cirurgia. ${ }^{(3)}$ Contudo, os procedimentos de CVL para níveis de miopia mais elevados são associados com maior enfraquecimento da córnea. Ainda, tipicamente, nos casos de ametropia mais negativa que - 8 a -12, observa-se previsibilidade refrativa reduzida, além de maior risco de perda da qualidade visual. ${ }^{(2)}$

Procedimentos refrativos intraoculares podem ser considerados, como o implante de lentes intraoculares fácicas (LIOf ou pIOL) e a cirurgia refrativa da catarata. A extração de cristalino transparente leva à perda de acomodação em pacientes jovens e aumenta o risco de descolamento de retina e maculopatias, não sendo recomendada para jovens altos míopes. ${ }^{(3)}$ É fundamental uma avaliação individualizada de cada candidato à cirurgia refrativa, para determinar qual o procedimento teria mais chances de sucesso. ${ }^{(4)}$

A principal indicação das lentes fácicas é a correção de altas ametropias, porém essas lentes podem ser utilizadas como adjuvantes no tratamento de ceratocone, pós-transplante de córnea, presbiopia e correção residual em pacientes pseudofácicos e após cirurgias a laser. Podem ser também consideradas em casos selecionados no grupo pediátrico, para correção de anisometropias e prevenção ou tratamento coadjuvante de ambliopia. ${ }^{(5)}$

\section{TIPOS DE LENTES INTRAOCULARES FÁCICAS}

As lentes fácicas podem sem classificadas de acordo com a localização do implante: na câmara anterior (anterior à íris) ou na câmara posterior (atrás da íris). As lentes de câmara anterior ainda podem ser subdivididas em de suporte angular ou fixação iriana.

As primeiras lentes fácicas foram desenhadas para serem implantadas na câmara anterior e apoiadas no ângulo camerular. Em 1953, Strampelli(6) implantou a primeira lente fácica para a correção de miopia, tendo sido associada a complicações graves, como descompensação endotelial e ovalização pupilar por fibrose do ângulo, as quais foram atribuídas ao material da lente e à medida inapropriada do tamanho do implante. A partir de então, diversos tipos de lentes foram produzidos, a fim de encontrar um material mais biocompatível e métodos mais confiáveis de aferir o tamanho ideal da lente a ser implantada.

Existem algumas opções de lentes fácicas disponíveis no mercado mundial. Muitas vezes, a mesma lente é comercializada em diferentes locais com nomes distintos, como Artisan ${ }^{\circledR}$, na Europa, e Verisyse ${ }^{\mathrm{TM}}$, nos Estados Unidos.

As principais alternativas de lentes à disposição do cirurgião estão dispostas na tabela 1.

Tabela 1. Lentes fácicas e suas características

\begin{tabular}{|c|c|c|c|c|c|}
\hline Localização & Nome & Marca & Material & $\begin{array}{c}\text { Tamanho } \\
\text { da incisão } \\
\text { (mm) }\end{array}$ & $\begin{array}{c}\text { Necessidade } \\
\text { de } \\
\text { iridectomia }\end{array}$ \\
\hline \multirow{2}{*}{ 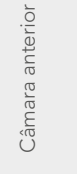 } & Artisan $®$ & $\begin{array}{l}\text { Ophthec } \\
\text { (Holanda) }\end{array}$ & PMMA & $5-6$ & Sim \\
\hline & Artiflex $®$ & $\begin{array}{l}\text { Ophthec } \\
\text { (Holanda) }\end{array}$ & $\begin{array}{c}\text { Ótica: } \\
\text { polisiloxana } \\
\text { Háptica: PMMA }\end{array}$ & 3 & Sim \\
\hline \multirow{2}{*}{ 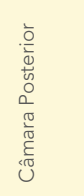 } & $\begin{array}{l}\text { Visian } \\
I^{\prime C L^{T M}}\end{array}$ & $\begin{array}{c}\text { STAAR } \\
\text { Surgical } \\
\text { (Estados } \\
\text { Unidos) }\end{array}$ & Collamer & 3,2 & $\begin{array}{l}\text { ICL 4b: sim } \\
\text { ICL 4c/V5: } \\
\text { não }\end{array}$ \\
\hline & $\begin{array}{l}\text { Eyecryl } \\
\text { Phakic }\end{array}$ & $\begin{array}{l}\text { Biotech } \\
\text { Visioncare } \\
\text { (Índia) }\end{array}$ & $\begin{array}{c}\text { Acrilíco } \\
\text { hidrofílico }\end{array}$ & 2,8 & Não \\
\hline
\end{tabular}

PMMA: polimetilmetacrilato.

No Brasil, conforme resolução do Conselho Federal de Medicina (CFM), processo-consulta CFM 4.296/2007, parecer CFM n.ㅇ/o8 (https://sistemas.cfm.org.br/normas/ arquivos/pareceres/BR/2008/6_2008.pdf), os implantes de lentes intraoculares de fixação iriana foram aprovados para correção de altas ametropias (miopia e hipermetropia) e afacias em abril de 2008. Em dezembro de 2018, foi divulgado parecer sobre o uso de lentes intraoculares fácicas de câmara posterior em pacientes com erros refrativos. Diz seu trecho de conclusão: "Para as finalidades deste pleito, consideramos adequada a pretensão do demandante pela equiparação da LIO fácica de câmara posterior às LIO fácicas de câmara anterior já reconhecidas pelo CFM na Resolução CFM no 1.843/2008" (https:// 
sistemas.cfm.org.br/normas/arquivos/pareceres/ BR/2018/38_2018.pdf). Desse modo, no Brasil, as lentes de câmara anterior de fixação iriana (Artisan ${ }^{\circledR}$ e Artiflex ${ }^{\circledR}$ ) e as de câmara posterior fácicas (ICL e Eyecryl) já têm seu uso liberado pelo CFM.

As lentes de fixação iriana de polimetilmetacrilato (PMMA) foram primeiramente desenvolvidas para a correção da afacia em 1977 pelo inovador holandês, Professor Jan Worst. ${ }^{(7)}$ Atualmente, são produzidas e distribuídas pela Ophtec BV (Groningen, Holanda) e comercializadas nos Estados Unidos pela Abbott Medical Optics (Illinois, Estados Unidos). No Brasil, é comercializada pelo nome Artisan ${ }^{\circledR}$ e a produzida nos Estados Unidos ganhou o nome de Verisyse ${ }^{\mathrm{TM}}$. Está disponível na faixa de dioptrias de -3,00 a -23,0D $\left(\right.$ Artisan $^{\circledR}$ ) e -5,0 a -20,0 D (Verisyse ${ }^{\mathrm{TM}}$ ). Adicionalmente, a Artisan ${ }^{\circledR}$ possui uma versão tórica disponível com poder de correção de até 7,5D cilíndricas. É uma lente de peça única em PMMA, não dobrável. Seus hápticos possuem uma pequena falha, dentro da qual o encravamento da média periferia do estroma iriano é realizado. Essa porção da íris é uma área virtualmente imóvel, entre a raiz da íris e a borda pupilar. Por ser uma área menos reativa e vascularizada, é esperado que suporte a pressão exercida pelas garras da lente, sem inibir a função iriana. Seu desenho propicia uma ótima distância tanto do endotélio quanto do cristalino, e a fixação na íris garante centralização estável. Todas as lentes têm diâmetro de $8,50 \mathrm{~mm}$. Esse tamanho único configura uma vantagem em relação às lentes que necessitam de adequada avaliação pré-operatória das dimensões das estrutras intraoculares. Possuem dois modelos que diferem no tamanho da parte óptica: modelo 206 (óptica 5,0mm, disponível de -1,00 a -25,50 D) e modelo 204 (óptica de 6mm, disponível de -1,00 a -15,50D). Dessa forma, a incisão para o implante da lente varia conforme o modelo escolhido: $5 \mathrm{~mm}$ ou $6 \mathrm{~mm}$. A versão tórica está disponível apenas no tamanho de $5 \mathrm{~mm}$.

A lente de fixação iriana dobrável disponível é a Artiflex ${ }^{\circledR}$, fabricada pela Ophtec BV. Ainda está em fase de análise clínica pelo Food and Drug Administration (FDA), com o nome de Veriflex (Abbott Medical Optics, Inc). Possui ótica de $6 \mathrm{~mm}$ de diâmetro em silicone e hápticos de PMMA, com comprimento total de $8,5 \mathrm{~mm}$, sendo implantada por incisão de 3,2mm. Está disponível nas dioptrias de -2,00 a -14,50D. Possui uma versão tórica (-1,00 a -13,50D, combinados com correção cilíndrica de -1,00 a $-5,00 D)$ que ainda não é comercializada no Brasil.

A primeira lente de câmara posterior foi desenvolvida em 1986 pelo inovador russo Svyatoslav Fyodorov, ${ }^{\left({ }^{(8)}\right.} \mathrm{e}$ somente os hápticos da lente ficavam atrás da íris, com a ótica da lente na câmara anterior. Foi relacionada a diversas complicações, como catarata e reações inflamatórias intensas em câmara anterior, tendo seu uso descontinuado. Atualmente, o modelo mais difundido para lentes fácicas de implante em câmara posterior é a Implantable Collamer $^{\circledR}$ Lens (ICL), fabricado pela STAAR ${ }^{\circledR}$ Surgical (Monrovia, CA, Estados Unidos). Desde que foi lançada, em 1993, seus modelos têm sido aperfeiçoados, e, atualmente, as versões V4 (V4b e V4c) e V5 (EVO+Visian ICL ${ }^{\mathrm{TM}}$ ) são as mais modernas utilizadas. O material da lente é descrito como Collamer ${ }^{\circledR}$, um polímero biocomopatível à base de hidroximetil metacrilato e colágeno, material exclusivo da STAAR ${ }^{\circledR}$ Surgical. O modelo V4 possui as versões V4b (Visian ICL ${ }^{\mathrm{TM}}$ ) e V4c (EVO Visian ICL ${ }^{\mathrm{TM}}$ ), que diferem quanto à presença do orifício central (KS-AquaPORT) na segunda, de $360 \mu \mathrm{m}$. A zona óptica do modelo V4C varia de 4,9 a 5,8mm enquanto o modelo $V 5$ possui zona ópticas maiores $(5,0$ a 6,1$)$. Ambas as lentes possuem o KS-AquaPORT. A presença dessa abertura central na lente elimina a necessidade de iridotomia ou iridectomia, que era obrigatória nos modelos anteriores. ${ }^{(3)}$ Permite, ainda, um fluxo de humor aquoso suficiente da câmara posterior para a anterior, mantendo a fisiologia normal do segmento anterior do olho. ${ }^{(3)} \mathrm{O}$ fato de dispensar a manipulação da íris assegura um tempo cirúrgico menor e diminui efeitos adversos provenientes do procedimento. ${ }^{(4)} \mathrm{A}$ versão tórica dessa lente não possui o orifício central e está disponível com cilindro de $+0,5$ a $+6,0 D$. As lentes para hipermetropia também não possuem o KS-AquaPORT e não estão disponíveis no Brasil.

A Eyecryl Phakic (Biotech Vision Care, Ahmedabad, Índia) é uma lente de câmara posterior asférica implantada através de uma incisão de 2,8 mm. Fabricada em acrílico hidrofílico, com ótica que varia de 4,65 a 5,50mm e faixa de dioptria entre -3,00 a -23,0oD. Possui versão tórica na faixa de 0,5 a 5D cilíndricas e também orifício central de $320 \mu \mathrm{m}$, que permite fluxo de humor aquoso adequado e dispensa a realização de iridectomia perioperatória. Apresenta quatro tamanhos diferentes: 12,omm, 12,5mm, $13,0 \mathrm{~mm}$ e $13,5 \mathrm{~mm}$.

\section{AVALIAÇÃO PRÉ-OPERATÓRIA}

A abordagem individualizada inclui desde uma anamnese bem feita para entender as motivações, necessidades e expectativas de cada caso durante exame oftalmológico completo, até exames complementares em uma abordagem propedêutica multimodal, que tem como propósito determinar a melhor indicação e o planejamento cirúrgico. 
Alguns critérios gerais devem ser seguidos quanto à indicação do implante de lentes fácicas. A idade maior que 18 a 21 anos é um fator a ser considerado, mas casos pediátricos também podem ser indicados para LIOf. Deve ser observada a ausência de catarata ou de outra doença intraocular, como uveíte, glaucoma ou desordens retinianas. Além disso, olhos com elevada ametropia podem apresentar progressão miópica em todas as fases da vida, ${ }^{(3)}$ e a estabilidade do erro refrativo deve ser observada por, pelo menos, 6 meses. Finalmente, condições que podem alterar a cicatrização, como gravidez e amamentação, contraindicam temporariamente a realização da cirurgia.

Deve ser realizado um exame oftalmológico completo que inclua medidas da acuidade visual com e sem correção, refração dinâmica e estática, biomicroscopia à lâmpada de fenda, tonometria e mapeamento de retina. Atenção especial deve ser dada a esse último exame, que deve ser realizado com identação escleral por oftalmologista treinado em retina e vítreo. Isso porque a maioria dos pacientes são altos míopes, com risco aumentado de roturas e descolamento de retina. Para pacientes que utilizam lente de contato, o uso deve ser descontinuado por, pelo menos, 7 dias antes do exame oftalmológico. Em pacientes usuários de lente de contato tóricas e rígidas, o uso deve ser descontinuado por período mais longo, dada a maior incidência de warpage e de instabilidade refracional. ${ }^{(3)}$ Deve haver documentação da estabilidade refracional, que pode ser considerada quando não há mudança maior que 0,5D num período de 6 meses a 1 ano. Exames complementares incluem paquimetria corneana, ceratometria (KRT), microscopia especular e medida do comprimento axial.

Componente importante para um implante seguro da lente intraocular é a medida da profundidade da câmara anterior (ACD), a qual pode ser obtida de imagens de ultrassom, imagens de Scheimpflug ou tomografia de coerência óptica. Essas técnicas demonstram resultados semelhantes em estudos comparativos. ${ }^{(5,9)}$ Existe correlação entre câmara anterior menor que 3,omm (medida a partir do epitélio corneano) e aumento de perda endotelial em pacientes com implante de lentes de fixação iriana Artisan ${ }^{\circledR} .^{(10,11)}$ Dessa forma, de maneira geral, a maioria das lentes exige ACD mínima de 3,omm a 3,2mm. Nos casos das lentes de câmara posterior, o valor mínimo é de 2,8 mm medidos a partir do endotélio.

Outro exame de fundamental importância é a microscopia especular com avaliação criteriosa da densidade e da morfologia das células endoteliais. O implante de LIOf tem risco potencial de perda endotelial relacionada tanto ao ato cirúrgico, de forma aguda, quanto à presença da lente, de forma crônica. Todas as lentes disponíveis possuem contagem endotelial mínima recomendada pré-operatória, que está relacionada à idade do paciente. Respeitar esses limites se torna essencial, na medida que essas lentes normalmente são implantadas em pacientes mais jovens, sendo importante preservar a densidade endotelial mínima, já que a perda induzida cirurgicamente é somada à perda fisiológica da idade. ${ }^{(3)}$ No Brasil, o valor mínimo preconizado pela resolução do CFM (https://sistemas.cfm.org.br/normas/arquivos/pareceres/ BR/2008/6_2008.pdf) que regulariza o uso das lentes de fixação iriana é de 2.000 células $/ \mathrm{mm}^{2}$.

O cálculo do poder da lente intraocular é realizado de acordo com o nomograma disponibilizado por cada fabricante.

As lentes de câmara posterior, como Visian ICL ${ }^{\mathrm{TM}} \mathrm{e}$ Eyecryl, possuem lentes com diferentes medidas de diâmetro total, de forma a acomodar as variações normais da anatomia intraocular. A Visian ICL ${ }^{\mathrm{TM}}$ oferece lentes com 12,1mm, 12,6mm, 13,2mm e 13,7mm de diâmetro. A Eyecryl possui lentes de 12,0 mm, 12,5mm, 13,0mm e 13,5mm de diâmetro. A escolha adequada do tamanho da lente a ser implantada e sua relação com as dimensões das estruturas da câmara posterior são consideradas a principal origem das complicações desse tipo de lente.

A maioria das complicações está associada ao vault adquirido no pós-operatório. Vault é a distância entre a cápsula anterior do cristalino e a face posterior da lente. Uma lente muito grande vai ficar longe do cristalino, ou seja, com o vault alto, o que resulta em estreitamento da câmara anterior, aumento do risco de fechamento angular ou bloqueio pupilar. Uma lente muito pequena resulta em distância pequena entre a lente e a cápsula anterior do cristalino, sendo o vault possivelmente insuficiente, o que pode gerar contato mecânico (principalmente na periferia das lentes divergentes, que são mais espessas) e aumentar o risco de formação de catarata. ${ }^{(12-14)}$

A seleção do tamanho da lente é, assim, o maior desafio que o cirurgião encontra no pré-operatório. Calculadoras on-line são fornecidas pelos fabricantes. A STAAR ${ }^{\circledR}$ Surgical (https://ocos.staarag.ch/) e Biotech (http://www.biotechcalculators.com// têm sua calculadora baseada na medida do branco a branco (WTW) horizontal, ACD, KRT e refração do paciente. A medida do WTW seria uma maneira de estimar o diâmetro do sulco ciliar, no qual a lente ficará posicionada. O diâmetro WTW pode ser aferido tradicionalmente por compassos manuais ou diversos aparelhos, como: Orbscan (Baush \& Lomb, Rochester, 
NY, Estados Unidos), IOL Master (Carl Zeiss Meditec, Jena, Alemanha), Pentacam (Oculus, Irvine, CA, Estados Unidos) e Lenstar (Haag Streit, Koeniz, Suíça). Os compassos manuais podem ser usados para validar medidas automatizadas, ajudando a detectar medidas erradas devido a alterações na área limbar, como arco senil, pigmentação, pinguécula e neovascularização - essa última sendo particularmente importante em usuários de lente de contato de longa data, como grande parte dos altos míopes. ${ }^{(4)}$

Porém, muitos estudos têm demonstrado fraca correlação entre a medida do WTW e o diâmetro do sulco ciliar, e, nos últimos anos, alguns cirurgiões e pesquisadores têm sugerido o uso de diferentes fórmulas que levam em consideração outras medidas que seriam relacionadas de maneira mais significativa ao diâmetro do sulco ciliar. ${ }^{(15-17)}$

Dougherty et al. ${ }^{(18)}$ desenvolveram um nomograma baseado na medida do sulco a sulco (STS) obtida da biomicroscopia ultrassônica e no poder da lente. Dos 73 olhos de 48 pacientes, nenhum teve vault menor que 9o $\mu \mathrm{m}$ (valor considerado como limite inferior e inadequado pelo estudo) ou maior que $1.000 \mu \mathrm{m}$ (considerado excessivo).O vault ficou entre $100 \mu \mathrm{m}$ e $500 \mu \mathrm{m}$ em 55 olhos $(76,6 \%)$ e entre $100 \mu \mathrm{m}$ e $700 \mu \mathrm{m}$ em 67 olhos (93,1\%). Comparando com o tamanho que teria sido escolhido, caso a medida da lente tivesse sido calculada usando o WTW, como recomendado pelo fabricante e aprovado pelo FDA, o método WTW teria recomendando lente maior em 47 (65,3\%) olhos, a mesma lente em 22 (30,6\%) e uma lente menor em $3(4,2 \%)$ casos. Choi et al. ${ }^{(19)}$ encontraram melhores valores de vault quando o comprimento da lente era determinado usando ultrassom de alta frequência (UBM) do que usando convencionalmente valores de WTW. Reportaram que o vault da lente estava adequado (no estudo definido entre $250 \mu \mathrm{m}$ e $750 \mu \mathrm{m}$ ) em todos os 13 (100\%) olhos quando o tamanho da lente era calculado pelo STS por meio de UBM. No grupo em que a lente foi calculada pelo WTW, esse valor foi adequado em apenas 53\% (9/17) dos olhos. Reinstein et al. ${ }^{\left({ }^{16}\right)}$ também demonstraram bons resultados medindo o STS e ângulo a ângulo (ATA) por meio de imagens obtidas pelo Artemis 1 (ArcScan Inc, Morrison, CO, Estados Unidos), um ultrassom digital de frequência muito alta (VHF), que, ao produzir imagens de maior qualidade, aumenta a reprodutibilidade dessas medidas.

Kojima et al. ${ }^{(12)}$ utilizaram a biomicroscopia ultrassônica e propuseram uma nova variável para o cálculo do tamanho da lente. A distância entre o plano do STS e a face anterior do cristalino foi chamada de sulcus to sulcus lens rise (STSL) e foi um parâmetro estatisticamente útil para aumentar a previsibilidade do vault adquirido no pós-operatório. Uma equação baseada nas medidas de STS, ACD e STSL foi proposta para chegar ao tamanho ideal da ICL. A fórmula calcula o tamanho ideal da lente a ser implantada para se atingir vault ideal de $500 \mu \mathrm{m}$. Por meio dessa nova equação, dos 81 olhos, nenhum teve vault insuficiente (0\%), 72 (88,9\%) obtiveram vaults moderados $(150 \mu \mathrm{m}$ a $1.000 \mu \mathrm{m})$ e nove $(11,1 \%)$ vaults altos (maior que $1,0 \mu \mathrm{m}$ ).

A partir da observação das estruturas nas imagens obtidas pelo UBM, Malyugin et al. ${ }^{(20)}$ verificaram que a periferia da camada pigmentada da íris correspondia à borda lateral do sulco ciliar e, apesar do OCT de câmara anterior não conseguir visualizar o sulco ciliar, retratava com boa qualidade imagens do fim da camada pigmentada da íris. Foi criado, então, um algoritimo baseado nessa distância entre fim do pigmento iriano (PTP) nasal e temporal, com boa repetitividade e baixa variabilidade.

Nakamura et al. ${ }^{(21)}$ desenvolveram uma fórmula baseada em medidas obtidas de forma automatizada por um tomógrafo de coerência óptica de câmara anterior (CASIA2; Tomey Corp, Nagoia, Japão), que utiliza parâmetros como a distância entre os esporões esclerais e o crystalline lens rise (CLR), que corresponde à distância entre o polo anterior do cristalino e a linha que mede o diâmetro horizontal da câmara anterior (linha entre o recesso angular nasal e temporal). Baseado nessas medidas, uma equação foi formulada, por meio de análise regressiva, e o valor ideal da ICL pode ser calculado. O valor obtido nem sempre é um o exato da lente disponível (ICL tem quatro tamanhos diferentes), sendo selecionada a lente maior mais próxima do valor obtido, o que pode explicar o grande número de vaults altos aquiridos. Por essa fórmula, dos 35 olhos estudados, 25 tiveram vault moderado, dois vaults insuficientes e oito vaults aumentados (valores de referência entre $250 \mu \mathrm{m}$ e $100 \mu \mathrm{m})$. Também foi elaborada uma fórmula capaz de predizer o vault esperado após a cirurgia e a diferença entre o vault pretendido, e o vault obtido no pós-operatório de 3 meses não apresentava diferença estatisticamente significativa.

Recentemente Reinstein (unpublished data, 2020, resultados apresentados no Simpósio Militar de Cirurgia Refrativa nos Estados Unidos, em 7 a 10 de janeiro de 2019. https://www.youtube.com/watch?v=-MvzluL_y_E) melhorou os resultados dos vaults obtidos incorporando as fórmulas de Kojima et al. ${ }^{(12)}$ e Dougherty et al., ${ }^{(18)}$ porém obtendo valores de STSL por meio de um ultrassom de frequência muito alta (ArcScan Insight ${ }^{\circledR} 100$ ), que captura as imagens de forma automatizada, gerando imagens de excelente resolução. Por meio de análise de regressão 
múltipa, foi possível selecionar variáveis relevantes para obtenção de um vault ideal: poder da ICL, STSL, diâmetro interno do corpo ciliar (CBID) - medido pelo ultrassom de muito alta frequência - e tamanho pupilar escotópico. Criou-se, então, uma equação capaz de calcular o vault que seria obtido se cada um dos tamanhos de ICL fosse implantado - e não mais o valor de lente ideal para cada olho. Assim, o cirurgião seria capaz de prescrever um vault determinado, levando em consideração fatores como a idade. Em pacientes mais jovens, seria possível escolher uma lente que gerasse vault mais alto, já que o vault tende a mudar com a idade e o crescimento fisiológico do cristalino $(25 \mu \mathrm{m}$ ao ano), proporcionando vault seguro durante todo o período de permanência da lente intraocular. Também deve ser considerada a ametropia a ser corrigida, já que pacientes mais míopes recebem lente com poder maior, e as lentes divergentes possuem bordas mais espessas que o centro. Portanto, um vault central próximo ao limite inferior aceitável poderia gerar um toque periférico da lente no cristalino e indução de catatara. Essa calculadora está disponível on-line no endereço iclsizing. com. Lee et al. também encontraram correlação entre diâmetro pupilar e vault pós-operatório, porém utilizaram o WTW para cálculo da ICL. Pupilas pequenas no pré-operatório estavam associadas a vaults menores $(250 \mu \mathrm{m}$ a $450 \mu \mathrm{m})$ em relação a pacientes do grupo de vaults médios $(451 \mu \mathrm{m}$ a $55 \mu \mathrm{m})$. Acreditam que pupilas menores possam exercer maior pressão sobre a ICL do que pupilas maiores, consequentemente diminuindo o vault. ${ }^{(14)}$

Em 2016, metanálise realizada por Packer demonstrou alto índice de similaridade no vault adquirido, independentemente do método utilizado na medida (WTW, STS, STSL e PTP), e intervalo adequado nos vaults adquiridos na maioria dos pacientes. Foi encontrada variação de $370 \mu \mathrm{m}$ a $640 \mu \mathrm{m}$ de vault para medidas pré-operatórias baseadas no STS e variação de $322 \mu \mathrm{m}$ a $594 \mu \mathrm{m}$ para medidas baseadas no WTW/ACD. O vault adquirido baseado nas medidas de STS e WTW não apresentou diferença estatística significativa. ${ }^{(2)}$

A Tabela 2 reúne alguns estudos e seus respectivos resultados em relação ao vault obtido por diferentes técnicas para medida do comprimento da ICL.

Em pacientes em que o erro refrativo ultrapassa o poder corretivo das lentes disponíveis ou para a correção de um eventual grau residual significativo, pode-se utilizar a combinação das técnicas lente fácica + Lasik. Essa combinação foi primeiramente descrita por Zaldivar et al. ${ }^{(24)}$ e denominada "bioptics". Inicialmente apresentada em associação com o Lasik, pode-se, porém, utilizar outras técnicas, como PRK e até mesmo incisões relaxantes. As cirurgias devem ser feitas com intervalos que podem variar de 3 a 12 meses, levando em consideração o tamanho da incisão corneana, a existência de suturas e a estabilidade refracional.

Tabela 2. Comparação dos resultados de vault obtidos e técnicas utilizadas para cálculo do comprimento da Implantable Collamer® Lens

\begin{tabular}{|c|c|c|c|c|}
\hline Estudos & $\begin{array}{l}\mathrm{n} \text { olhos/ } \\
\text { pacientes }\end{array}$ & $\begin{array}{l}\text { Parâmetro } \\
\text { utilizado }\end{array}$ & $\begin{array}{l}\text { Aparelho utilizado } \\
\text { para medida }\end{array}$ & $\begin{array}{c}\text { Vault médio } \pm \\
\text { DP (faixa) }\end{array}$ \\
\hline Kojima et al..$^{(12)}$ & $81 / 43$ & STS/STSL & $\begin{array}{l}\text { UBM VuMAX'M } \| \\
\text { High Frequency UBM } \\
\text { (Sonomed, Inc) }\end{array}$ & $\begin{array}{l}640 \pm 250 \mu m \\
(190-1.330)\end{array}$ \\
\hline Reinstein et al. ${ }^{(17)}$ & $50 / 25$ & STS & Artemis 2 (ArcScan Inc) & $\begin{array}{c}370 \pm 160 \mu \mathrm{m} \\
(80-920)\end{array}$ \\
\hline Dougherty et al. ${ }^{(18)}$ & $73 / 73$ & STS & $\begin{array}{l}\text { UBM VuMAXTM ॥ } \\
\text { High Frequency UBM } \\
\text { (Sonomed, Inc) }\end{array}$ & $\begin{array}{c}340 \pm 174 \mu \mathrm{m} \\
(90-952)\end{array}$ \\
\hline Malyugin et al. ${ }^{(20)}$ & $32 / 32$ & PTP & $\begin{array}{c}\text { OCT-CA Visante (Carl } \\
\text { Zeiss) }\end{array}$ & $\begin{array}{l}530 \pm 180 \mu \mathrm{m} \\
(240-840)\end{array}$ \\
\hline Nakamura et al. ${ }^{(21)}$ & $35 / 18$ & ACW/CLR & $\begin{array}{l}\text { OCT-CA CASIA2 } \\
\text { (Tomey Corp.) }\end{array}$ & $\begin{array}{l}785 \pm 271 \mu \mathrm{m} \\
(193-1.308)\end{array}$ \\
\hline Yan et al. ${ }^{(22)}$ & $61 / 32$ & WTW/ACD & Pentacam (Oculus) & $\begin{array}{c}449 \pm 167 \mu \mathrm{m} \\
(130-810)\end{array}$ \\
\hline Igarashi et al. ${ }^{(23)}$ & $44 / 23$ & WTW & $\begin{array}{c}\text { OCT-CA CASIA2 } \\
\text { (Tomey Corp.) }\end{array}$ & $\begin{array}{c}491 \pm 189 \mu \mathrm{m} \\
(117-995)\end{array}$ \\
\hline
\end{tabular}

\section{LENTES FÁCICAS EM SITUAÇÕES ESPECIAIS}

As lentes fácicas podem ser usadas em algumas situações especiais como adjuvantes ao tratamento de outras doenças. ${ }^{(25)}$

Pacientes com ceratocone podem se beneficiar do implantes dessas lentes. Denominada Bioptics Terapêutico, ${ }^{(26)}$ a associação de lentes fácicas com o implante de anel intraestromal (Figura 1), ou crosslinking, vem sendo discutida como alternativa para pacientes com ceratocone estável. O implante de anel intraestromal melhora a topografia da córnea, reduzindo o astigmatismo irregular e algumas aberrações de alta ordem, porém muitos olhos com ceratocone apresentam também alta miopia. Estudos recentes demonstram que o implante de lentes fácicas nesses pacientes constituiu maneira efetiva, segura e previsível de corrigir a alta miopia desses pacientes. ${ }^{(27-29)}$

Alguns autores têm estudado a eficácia das lentes fácicas para a correção de altas ametropias em pacientes pediátricos com ambliopia por anisometropia. Pacientes com anisometropia maior que 3D são menos tolerantes ao uso de óculos, devido à aniseconia importante e à diplopia. Zhang et al. ${ }^{(30)}$ implantaram lente fácica de câmara posterior (ICL) em 11 olhos diagnosticados com ambliopia por alta miopia unilateral e relataram melhora significativa da acuidade visual e sensibilidade ao contraste. 


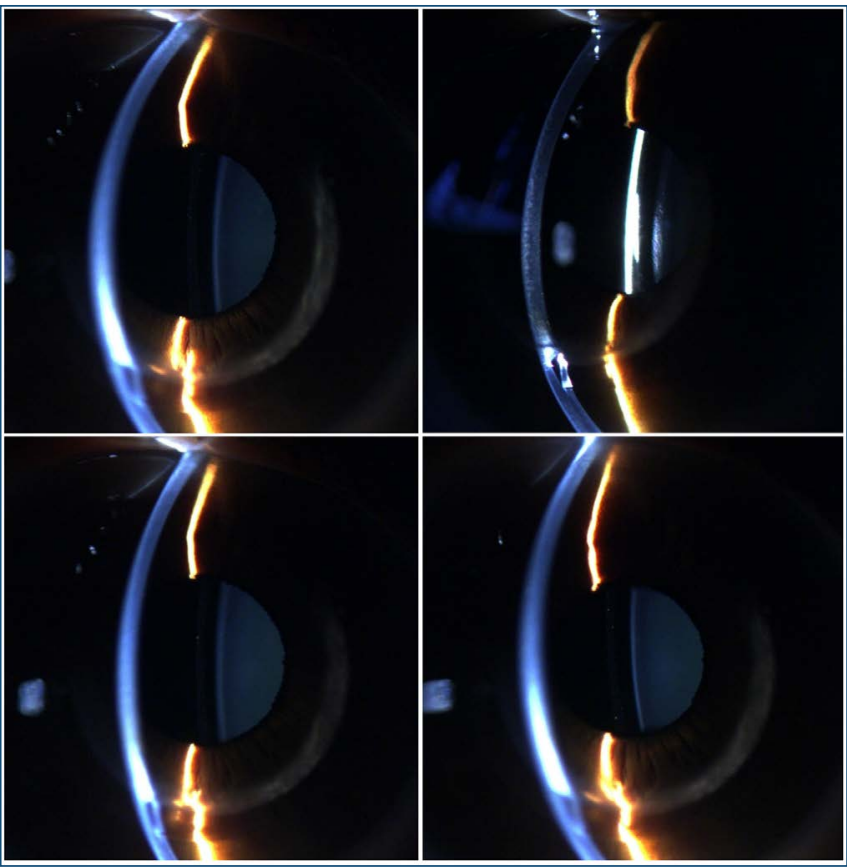

onte: cortesia do Dr. Renato Ambrósio Júnior.

Figura 1. Bioptics terapêutico. Associação de lente fácica com anel intraestromal em paciente com ceratocone.

Lentes fácicas também pode ser ser alternativa viável para o tratamento de erros refrativos significativos após ceratoplastia penetrante ${ }^{(31)}$ e para a correção de ametropias residuais significativas e/ou sintomáticas em pacientes pseudofácicos. ${ }^{(32)}$

\section{COMPLICAÇÕES PÓS-OPERATÓRIAS}

As complicações com as lentes fácicas podem ser inferidas de acordo com sua localização no olho: quanto mais próxima a relação da lente com o endotélio corneano, a íris ou o cristalino, maior a chance de perda de células endoteliais, complicações irianas e catarata, respectivamente.

Lentes que exigem a realização de iridectomia ganham adicional risco de complicações decorrentes do procedimento, como imagens secundárias, hifema, catarata localizada e sinéquias. ${ }^{(4)}$

As lentes de fixação iriana preocupam em relação à perda de células endoteliais a longo prazo. Alguns estudos mostram correlação negativa entre a ACD e perda de células endoteliais a longo prazo, correlação que permaneceu após 7 anos de seguimento, ${ }^{(33)}$ sugerindo, inclusive, que os critérios mínimos para a realização da cirurgia propostos pelo FDA (ACD de 2,6mm e CE de 2.000 cels $/ \mathrm{mm}^{2}$ ) sejam vistos com cautela, principalmente em pacientes jovens com valores de ACD e CE no limite inferior. Jonker et al. ${ }^{(10)}$ demonstraram perda endotelial crônica de $16,6 \%$ e $21,4 \%$ de 6 meses a 10 anos em pacientes do grupo miópico e grupo tórico, respectivamente. Nesse mesmo estudo, a taxa de explante das lentes intraoculares devido à perda endotelial foi de $6,0 \%$ e 4,8\% nos grupos miópico e tórico, respectivamente. A Agence Française de Sécurité Sanitaire et des Produits de Santé (AFSSAPS) sugere um ponto de corte para explante da pIOL: perda maior que 30\% em relação a CE pré-operatória ou CE menor que 1.500cels $/ \mathrm{mm}^{2}$.(34)

Em relação às lentes de câmara posterior, a complicação que mais merece atenção é a formação de catarata. De acordo com metanálise de Packer, ${ }^{(35)}$ modelos anteriores da ICL, como V4b, tinham probabilidade de 1,1\% a 5,9\% de ocorrência de catarata, e a probabilidade de precisarem de facectomia era de zero a 1,8\%. Outros fatores de risco encontrados foram idade maior que 40 anos, altas miopias (mais que -12,0, lembrando que a alta miopia, em si, é fator de risco para catarata, mesmo em pacientes sem cirurgia ocular prévia) e vault pós-operatório pequeno.

Entretanto, o surgimento dos novos modelos V4c e V5, que contam com o orifício central, melhorando a circulação do humor aquoso, aparentemente mudou a situação. Novos estudos ainda são necessários, pois, por serem modelos novos, o maior seguimento reportado tem 5 anos e número limitado de paciente, mas Shimizu et al. ${ }^{(36)}$ reportaram incidência nula de opacidade subcapsular anterior e catarata com o uso da lente Visian ICL ${ }^{\mathrm{TM}}$ V4C.

Em relação à perda de células endoteliais, as lentes de câmara posterior, considerando sua distância do endotélio, apresentam taxas menores de perda endotelial. Moya et al. ${ }^{(37)}$ publicaram estudo retrospectivo de 12 anos avaliando 144 olhos com implante de ICL e estimaram perda de 6,46\% induzida cirurgicamente no primeiro ano, além de taxa anual de 1,2\% após isso. Em estudo de seguimento de 8 anos, Igarashi et al. ${ }^{(38)}$ reportaram perda média de $6,2 \%$ após implante de ICL em 41 olhos de 41 pacientes. A lente Eyecryl está no mercado há menos tempo, e, assim, os estudo tem seguimento menor. Em estudo retrospectivo que envolvia 52 olhos de 26 pacientes, Urdem et al. ${ }^{(39)}$ relataram perda média de 4,51\% em 1 ano e 0,98\% entre o primeiro e segundo ano, resultando em perda cumulativa de 5,67\% $₫ 10,8 \%$ em 2 anos de acompanhamento pós-operatório. Apesar da perda ser menor quando comparada a outros tipos de lentes, a saúde endotelial dos pacientes candidatos ao implante de lente fácica de câmara posterior deve ser avaliada cuidadosamente.

Durante o seguimento o paciente com LIOf de câmara posterior, um dos aspectos clínicos a ser observado é o vault da lente intraocular. Esse valor, que corresponde à distância entre a cápsula anterior do cristalino e a face posterior da lente, é consequência, principalmente, da relação entre o tamanho da lente intraocular escolhida e as dimensões da câmara posterior do paciente. ${ }^{(40)}$ 


\section{RESULTADOS CLÍNICOS}

Altas ametropias, em especial a miopia, estão associadas à perda da qualidade vida e de autoestima dos pacientes. ${ }^{(41,42)}$ Desse modo, geralmente, pela melhora rápida da acuidade visual sem correção e manutenção da acomodação, a maioria dos pacientes reportam altos níveis de satisfação quando submetidos ao implante de LIO. Em estudo com 32 pacientes (61 olhos) que tiveram lente de câmara posterior implantada, Yan et al. ${ }^{(22)}$ construíram uma escala na qual zero correspondia a muito insatisfeito e dez a muito satisfeito e reportaram que "todos os pacientes estavam satisfeitos com sua performance visual, e $71 \%$ estavam mais que satisfeitos (muito satisfeitos)". A nota média de satisfação desses pacientes era 9,27士0,87. Todos responderam "sim" à pergunta: "Consideraria recomendar essa cirurgia a pacientes como você?".

Medidas de efetividade do procedimento incluem acuidade visual pós-operatória sem correção, equivalente esférico da refração manifesta no pós-operatório, previsibilidade refrativa (porcentagem de olhos que se mantiveram com o equivalente esférico da refração pós-operatória entre $\pm 0,5$ e $\pm 1,0$ ) e estabilidade refrativa. $O$ índice de eficácia da lente corresponde à razão entre a acuidade visual pós-operatória sem correção e a acuidade visual pré-operatória com correção. A segurança da lente leva em conta fatores como manutenção da acuidade visual com correção, mudanças na pressão intraocular, alterações da densidade de células endoteliais, opacidade subcapsular anterior ou catarata significativa e intervenções cirúrgicas secundárias. O índice de segurança corresponde à divisão entre a acuidade visual pós-operatória com correção e a acuidade visual pré-operatória com correção.

As lentes de fixação iriana possuem uma zona óptica maior que o Lasik, causando menos sintomas visuais, como glare e halos em pacientes com pupilas mesópicas maiores. ${ }^{(43)}$ Ensaios clínicos do FDA, num acompanhamento de 3 anos, mostraram acuidade visual sem correção de 20/40 ou melhor em $84 \%$ dos pacientes e acuidade visual com correção 20/40 ou melhor em 100\% dos pacientes (49\% melhoraram a acuidade visual com correção, 45\% não tiveram mudança e $6 \%$ perderam uma a duas linhas). Destes pacientes, 31\% obtiveram acuidade visual sem correção igual ou melhor que 20/20.(44) Quando comparado com Lasik para a correção de miopia entre -8 e -12, as lentes fácicas $\left(\right.$ Artisan $^{\circledR}$ ) obtiveram índice de segurança (relação entre acuidade visual com correção pós-operatória e acuidade visual com correção pré-operatória) superior e foram preferidas por mais pacientes. ${ }^{(45)}$
A lente de câmara posterior Visian ICL ${ }^{\mathrm{TM}}$, aprovada para uso nos Estados Unidos, demonstra altos índices de eficácia - maior que 1,0 em todos os casos analisados. ${ }^{(2)}$ Grande porcentagem dos casos atingiu e manteve acuidade visual igual ou melhor que 20/20. Shimizu et al. ${ }^{(36)}$ reportaram que a acuidade visual sem correção no grupo ICL foi maior ou igual a 20/20 em 1, 3 e 6 meses e em 1, 3 e 5 anos em 97\%, 100\%, 100\%, 100\%, 100\% e 85\% dos casos analisados, respectivamente. Quanto à mudança de aberrações de alta ordem e sensibilidade ao contraste, Igarashi et al., ${ }^{(46)}$ em estudo comparativo, notaram que, em olhos com miopia maior que -6D, os pacientes do grupo ICL tiveram significativamente menos mudanças nas aberrações de alta ordem em relação aos pacientes do grupo Lasik guiado por wavefront. Como no implante das lentes ICL não há alteração do formato da córnea, mudanças nessas aberrações são leves, e não há alteração da magnificação retiniana da imagem, o que faz com esses pacientes também apresentem aumento da sensibilidade ao contraste. ${ }^{(47)} \mathrm{Nas}$ cirurgias fotorrefrativas, como a com Lasik, a regressão a longo prazo foi reportada, a qual é tão mais frequente e debilitante quanto maior for a ametropia corrigida. ${ }^{(47)}$ Apesar do índice de segurança do Lasik também ser maior que 1,0 nos estudos avaliados, ${ }^{(48-50)}$ a previsibilidade estava entre $42 \%$ e $88 \%$, indicando o efeito da regressão. ${ }^{(49)} \mathrm{O}$ índice de retratamento também foi alto, de 18\% a 30,8\% nos estudos avaliados por Ikeda et al. ${ }^{(49)}$ A regressão, nesses casos de Lasik, parece estar relacionada ao aumento da curvatura corneana. No implante de lentes ICL, a estabilidade refracional a longo prazo é maior, principalmente nos modelos mais novos, que diminuíram sensivelmente o índice de catarata como complicação. ${ }^{(35)}$ Antes dos modelos com orifício central (Visian ICL ${ }^{\mathrm{TM}}$ V4C), o surgimento de opacidade de cristalino era importante causa de mudança na refração desses pacientes. No seguimento de 8 anos, Igarashi et al. demonstraram correlação significativa entre mudanças na refração e alterações no comprimento axial, o que pode indicar que o alongamento axial pode ser um dos fatores responsáveis pela instabilidade refracional após implante de ICL, principalmente em olhos maiores que $27,5 \mathrm{~mm} .{ }^{\text {(38) }}$

A versão tórica da ICL apresenta boa estabilidade, como demonstrado por Lee et al. Logo após a cirurgia, o grau de rotação absoluta foi de $2,81^{\circ} \pm 1,87^{\circ}$ e de $3,75^{\circ} \pm 2,92^{\circ}$

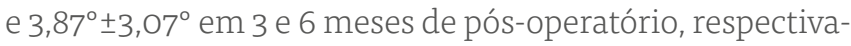
mente. O índice de eficácia foi de 1,35£0,19 e de segurança de 1,38 $\pm 0,22$, sendo alterantiva segura e eficiente na correção do astigmatismo. ${ }^{(51)}$

A tabela 3 compara resultados clínicos obtidos nos estudos avaliados. 
Tabela 3. Resultados clínicos

\begin{tabular}{|c|c|c|c|c|c|c|c|c|}
\hline Estudos & Lente & $\begin{array}{l}\mathrm{n} \text { olhos/ } \\
\text { pacientes }\end{array}$ & $\begin{array}{l}\text { Seguimento } \\
\text { (meses) }\end{array}$ & $\begin{array}{l}\text { EE pré-operatório } \\
\text { (LogMar) } \pm \text { DP }\end{array}$ & $\begin{array}{l}\text { Média AV pós- } \\
\text { operatório sem } \\
\text { correção (LogMar) } \\
\text { 土 DP }\end{array}$ & $\begin{array}{l}\text { Previsibilidade } \\
\%( \pm 0,5 / \pm 1,0)\end{array}$ & $\begin{array}{l}\text { Índice de } \\
\text { eficácia }\end{array}$ & $\begin{array}{l}\text { Índice de } \\
\text { segurança }\end{array}$ \\
\hline Yan et al. ${ }^{(22)}$ & $\mathrm{ICL}, \mathrm{V} 4 \mathrm{c}$ & $61 / 32$ & 24 & $-14,62 \pm 4,29$ & $+0,1 \pm 0,6$ & $79 / 98$ & 1,03 & 1,24 \\
\hline Shimizu et al..$^{(36)}$ & $\mathrm{ICL}, \mathrm{V} 4 \mathrm{c}$ & $64 / 32$ & 60 & $-7,54 \pm 2,4$ & $-0,17 \pm 0,14$ & $88 / 96$ & 1,0 & 1,15 \\
\hline $\begin{array}{l}\text { Kamiya et al. }{ }^{(52)} \text { (miopia leve a } \\
\text { moderada, } \mathrm{EE}>-6 \text { ) }\end{array}$ & $\mathrm{ICL}, \mathrm{V} 4 \mathrm{C}$ & $57 / 57$ & 12 & $-4,29 \pm 1,31$ & $-0,17 \pm 0,14$ & $93 / 98$ & NR & NR \\
\hline Kamiya et al. ${ }^{(52)}$ (miopia alta, EE $\leq-6$ ) & $\mathrm{ICL}, \mathrm{V} 4 \mathrm{c}$ & $294 / 294$ & 12 & $-10,13 \pm 2,64$ & $-0,16 \pm 0,09$ & $94 / 99$ & NR & NR \\
\hline Lee et al. ${ }^{(53)}$ & $\mathrm{ICL}, \mathrm{V} 4$ & $281 / 145$ & $61-110$ & $-8,74 \pm 2,27$ & $+0,02 \pm 0,19$ & $69,8 / 87,2$ & 1,2 & 1,04 \\
\hline \multirow[t]{2}{*}{ Kojima et al. ${ }^{(54)}$} & $\mathrm{ICL}, \mathrm{V} 4 \mathrm{c}$ & $23 / 46^{*}$ & 6 & $-7,32 \pm 3,42$ & $-0,23 \pm 0,9$ & $94,7 / 100$ & 1,16 & 1,21 \\
\hline & $\mathrm{ICL}, \mathrm{V} 5$ & $23 / 46^{\star}$ & 6 & $-7,09 \pm 2,84$ & $-0,19 \pm 0,12$ & $84,2 / 100$ & 1,03 & 1,19 \\
\hline Yaşa et al. ${ }^{(55)}$ & Eyecryl Phakic & $58 / 29$ & 12 & $-13,41 \pm 3,22$ & $+0,21 \pm 0,66$ & $62 / 93$ & 1,20 & 1,39 \\
\hline
\end{tabular}

Previsibilidade: porcentagem dos pacientes com EE entre $\pm 0,5$ e $\pm 1,0 \mathrm{D}$.

*23 pacientes implantaram ICL V4c em um olho e V5 no outro, total 46 olhos; valor aproximado, estudo relata AV em decimais: $+0,84 \pm 0,28$.

EE: equivalente esférico; DP: desvio-padrão; AV: acuidade visual; ICL: Implantable Collamer® Lens; NR: não reportado.

\section{CONCLUSÃO}

Desde o primeiro implante de lentes fácicas em 1953 por Strampelli, ocorreram mudanças significativas no desenho e no material das lentes. Diversos estudos com períodos longos de acompanhamento demonstram a segurança e a efetividade do uso dessa modalidade cirúrgica, que, apesar de exigir curva de aprendizado, é familiar à maioria dos cirurgiões de segmento anterior. Esses estudos fornecem dados para aumentar a segurança dos implantes, definindo limites seguros de parâmetros importantes e indispensáveis para o bom resultado cirúrgico, como contagem de células endotelias, profundidade de câmara anterior e, principalmente, medidas a serem usadas ao definir o tamanho da lente a ser implantada (no caso de lentes de câmara posterior), o que constitui o principal desafio para o cirurgião. Sabe-se do impacto que a alta miopia tem na qualidade de vida dos pacientes e, em muitos casos, por diversas razões, a cirurgia refrativa a laser não pode ser executada. O implante de lentes fácicas constitui opção segura e eficiente, associada a um ao alto índice de satisfação dos pacientes. Certamente, os benefícios suplantam os riscos inerentes ao procedimento, o qual deve ser considerado como alternativa à cirurgia fotorrefrativa corneana.

\section{REFERÊNCIAS}

1. Ambrósio R Jr. Cirurgia refrativa terapêutica: por que diferenciar? Rev Bras Oftalmol. 2013;72(2):85-6

2. Packer M. Meta-analysis and review: effectiveness, safety, and central port design of the intraocular collamer lens. Clin Ophthalmol. 2016;10:1059-77.

3. Kawamorita T, Uozato H, Shimizu K. Fluid dynamics simula.tion of aqueous humour in a posterior-chamber phakic intraocular lens with a central perforation. Graefes Arch Clin Exp Ophthalmol. 2012;250(6):935-9.

4. Huang D, Schallhorn SC, Sugar A, Farjo AA, Majmudar PA, Trattler WB, et al. Phakic intraocular lens implantation for the correction of myopia: a report by the American Academy of Ophthalmology. Ophthalmology. 2009;116(11):2244-58.

5. Frisch IB, Rabsilber TM, Becker KA, Reuland AJ, Auffarth GU. Comparison of anterior chamber depth measurements using Orbscan II and IOLMaster. Eur J Ophthalmol. 2007;17(3):327-31.

6. Strampelli B. Sopportabilita di lenti acriliche in camera anteriore nella afachia e nei vizi di refrazione. Ann Ottalmol Clin Ocul 1954;80:75-82.
7. Landesz M, Worst JG, Siertsema JV, van Rij G. Correction of high myopia with the Worst myopia claw intraocular lens. J Refract Surg. 1995;11(1):1625.

8. Fyodorov SN, Zuev VK, Tumanyan ER. Modern approach to the stagewise complex surgical therapy of high myopia. Transactions of International Symposium of IOL Implantation and Refractive Surgery. Moscow: RSFSP Ministry of Health;1987. p. 274-9.

9. Dada T, Sihota R, Gadia R, Aggarwal A, Mandal S, Gupta V. Comparison of anterior segment optical coherence tomography and ultrasound biomicroscopy for assessment of the anterior segment. J Cataract Refract Surg. 2007;33(5):837-40.

10. Jonker SM, Berendschot TT, Ronden AE, Saelens IE, Bauer NJ, Nuijts RM. Long-term endothelial cell loss in patients with artisan myopia and artisan toric phakic intraocular lenses: 5-and 10-year results. Ophthalmology. 2018;125(4):486-94.

11. Galvis V, Villamil JF, Acuña MF, Camacho PA, Merayo-Lloves J, Tello A, et al. Long-term endothelial cell loss with the iris-claw intraocular phakic lenses (Artisan®). Graefes Arch Clin Exp Ophthalmol. 2019;257(12):2775-87.

12. Kojima T, Yokoyama S, Ito M, Horai R, Hara S, Nakamura T, et al. Optimization of an implantable collamer lens sizing method using highfrequency ultrasound biomicroscopy. Am J Ophthalmol. 2012;153(4):6327, 637.e1.

13. Lee DH, Choi SH, Chung ES, Chung TY. Correlation between preoperative biometry and posterior chamber phakic Visian Implantable Collamer Lens vaulting. Ophthalmology. 2012;119(2):272-7.

14. Lee H, Kang DS, Choi JY, Ha BJ, Kim EK, Seo KY, et al. Analysis of preoperative factors affecting range of optimal vaulting after implantation of 12.6-mm V4c implantable collamer lens in myopic eyes. BMC Ophthalmol. 2018;18(1):163.

15. Pop M, Payette $Y$, Mansour M. Predicting sulcus size using ocular measurements. J Cataract Refract Surg. 2001;27(7):1033-8.

16. Reinstein DZ, Archer TJ, Silverman RH, Rondeau MJ, Coleman DJ. Correlation of anterior chamber angle and ciliary sulcus diameters with white-to-white corneal diameter in high myopes using artemis VHF digital ultrasound. J Refract Surg. 2009;25(2):185-94.

17. Reinstein DZ, Lovisolo CF, Archer TJ, Gobbe M. Comparison of postoperative vault height predictability using white-to-white or sulcus diameter-based sizing for the visian implantable collamer lens. J Refract Surg. 2013;29(1):30-5.

18. Dougherty PJ, Rivera RP, Schneider D, Lane SS, Brown D, Vukich J. Improving accuracy of phakic intraocular lens sizing using high-frequency ultrasound biomicroscopy. J Cataract Refract Surg. 2011;37(1):13-8.

19. Choi KH, Chung SE, Chung TY, Chung ES. Ultrasound biomicroscopy for determining visian implantable contact lens length in phakic IOL implantation. J Refract Surg. 2007;23(4):362-7.

20. Malyugin BE, Shpak AA, Pokrovskiy DF. Posterior chamber phakic intraocular lens sizing based on iris pigment layer measurements by anterior segment optical coherence tomography. J Cataract Refract Surg. 2015;41(8):1616-22. 
21. Nakamura T, Isogai N, Kojima T, Yoshida Y, Sugiyama Y. Implantable collamer lens sizing method based on swept-source anterior segment optical coherence tomography. Am J Ophthalmol. 2018;187:99-107.

22. Yan Z, Miao H, Zhao F, Wang X, Chen X, Li M, et al. Two-Year Outcomes of Visian Implantable Collamer Lens with a Central Hole for Correcting High Myopia. J Ophthalmol. 2018;2018:8678352.

23. Igarashi A, Shimizu K, Kato S, Kamiya K. Predictability of the vault after posterior chamber phakic intraocular lens implantation using anterior segment optical coherence tomography. J Cataract Refract Surg. 2019;45(8):1099-104.

24. Zaldivar R, Davidorf JM, Oscherow S, Ricur G, Piezzi V. Combined posterior chamber phakic intraocular lens and laser in situ keratomileusis: bioptics for extreme myopia. J Refract Surg. 1999;15(3):299-308.

25. Pineda R 2nd, Chauhan T. Phakic intraocular lenses and their special indications. J Ophthalmic Vis Res. 2016;11(4):422-8.

26. Ambrosio Júnior R. Intracorneal ring segment, phakic IOL implantation enable keratoconus management without keratoplasty. Ocul Surg News. 2011; Dec 10. [cited 2020 Nov 10]. Available from: https://www.healio. com/news/ophthalmology/20120331/intracorneal-ring-segment-phakiciol-implantation-enable-keratoconus-management-without-keratoplasty

27. Alfonso JF, Lisa C, Fernández-Vega L, Madrid-Costa D, Poo-López A, Montés-Micó R. Intrastromal corneal ring segments and posterior chamber phakic intraocular lens implantation for keratoconus correction. J Cataract Refract Surg. 2011;37(4):706-13.

28. Alio J, Sanz-Diez P. Phakic intraocular lenses in keratoconus. Int J Kerat Ect Cor Dis. 2015;4:103-6.

29. Güell JL, Elies D, Verdaguer P, Gris O, Manero F, Morral M. Phakic Intraocular Lenses in Keratoconus. Cataract. Berlim: Karger Publishers; 2013. p. 100-15.

30. Zhang J, Li JR, Chen ZD, Yu MB, Yu KM. Phakic posterior chamber intraocular lens for unilateral high myopic amblyopia in Chinese pediatric patients. Int J Ophthalmol. 2016;9(12):1790-7.

31. Fares $U$, Sarhan AR, Dua HS. Management of post-keratoplasty astigmatism. J Cataract Refract Surg. 2012;38(11):2029-39.

32. Chiou AG, Bovet J, de Courten C. Pseudophakic ametropia managed with a phakic posterior chamber intraocular lens. J Cataract Refract Surg. 2001;27(9):1516-8.

33. Saxena R, Boekhoorn SS, Mulder PG, Noordzij B, van Rij G, Luyten GP. Long-term follow-up of endothelial cell change after Artisan phakic intraocular lens implantation. Ophthalmology. 2008;115(4):608-613.e1.

34. Bernard P, Fournier M. Definitive stop of marketing, product recall and follow-up of implanted patients. Presbyopic Intraocular Lenses NewLife/ Vivarte Presbyopic: Agence Française de Sécurité Sanitaire et des Produits de Santé (AFSSAPS); 2007.

35. Packer M. The Implantable Collamer Lens with a central port: review of the literature. Clin Ophthalmol. 2018;12:2427-38.

36. Shimizu K, Kamiya K, Igarashi A, Kobashi H. Long-term comparison of posterior chamber phakic intraocular lens with and without a central hole (hole ICL and conventional ICL) implantation for moderate to high myopia and myopic astigmatism: consort-compliant article. Medicine (Baltimore). 2016;95(14):e3270.

37. Moya T, Javaloy J, Montés-Micó R, Beltrán J, Muñoz G, Montalbán R. Implantable collamer lens for myopia: assessment 12 years after implantation. J Refract Surg. 2015;31(8):548-56.

38. Igarashi A, Shimizu K, Kamiya K. Eight-year follow-up of posterior chamber phakic intraocular lens implantation for moderate to high myopia. Am J Ophthalmol. 2014;157(3):532-9.e1.
39. Urdem U, Agca A. Refractive results and endothelial cell density after eyecryl

phakic intraocular lens implantation. Beyoglu Eye J. 2019; 4(1):17-22.

40. Zeng $\mathrm{QY}, \mathrm{Xie} X \mathrm{~L}$, Chen $\mathrm{Q}$. Prevention and management of collagen copolymer phakic intraocular lens exchange: causes and surgical techniques. J Cataract Refract Surg. 2015;41(3):576-84.

41. Brar S. Incidence \& factors responsible for implantable collamer lens (ICL) explantation \& outcomes of fur-ther management-5 year retrospective study. EC Ophthalmology. 2015;3:231-9.

42. Rose K, Harper R, Tromans C, Waterman C, Goldberg D, Haggerty C, et al Quality of life in myopia. Br J Ophthalmol. 2000;84(9):1031-4.

43. Yesilirmak N, Davis Z, Yoo SH. Refractive Surgery (SMILE vs. LASIK vs. Phakic IOL). Int Ophthalmol Clin. 2016;56(3):137-47.

44. Stulting RD, John ME, Maloney RK, Assil KK, Arrowsmith PN, Thompson VM; U.S. Verisyse Study Group. Three-year results of Artisan/Verisyse phakic intraocular lens implantation. Results of the United States Food And Drug Administration clinical trial. Ophthalmology. 2008;115(3):464472.e1.

45. Malecaze FJ, Hulin H, Bierer P, Fournié $P$, Grandjean $H$, Thalamas $C$, et al. A randomized paired eye comparison of two techniques for treating moderately high myopia: LASIK and artisan phakic lens. Ophthalmology. 2002;109(9):1622-30.

46. Igarashi A, Kamiya K, Shimizu K, Komatsu M. Visual performance after implantable collamer lens implantation and wavefront-guided laser in situ keratomileusis for high myopia. Am J Ophthalmol. 2009;148(1):16470.e1.

47. Igarashi A. Posterior chamber phakic IOLs vs. LASIK: benefits and complications. Expert Rev Ophthalmol. 2019;14(1):43-52.

48. Alió JL, Ortiz D, Muftuoglu O, Garcia MJ. Ten years after photorefractive keratectomy (PRK) and laser in situ keratomileusis (LASIK) for moderate to high myopia (control-matched study). Br J Ophthalmol. 2009;93(10):13138.

49. Ikeda T, Shimizu K, Igarashi A, Kasahara S, Kamiya K. Twelve-year followup of laser in situ keratomileusis for moderate to high myopia. Biomed Res Int. 2017;2017:9391436.

50. Alió JL, Soria F, Abbouda A, Peña-García P. Laser in situ keratomileusis for -6.00 to -18.00 diopters of myopia and up to -5.00 diopters of astigmatism: 15-year follow-up. J Cataract Refract Surg. 2015;41(1):33-40.

51. Lee H, Kang DS, Choi JY, Ha BJ, Kim EK, Seo KY, et al. Rotational stability and visual outcomes of V4c toric phakic intraocular lenses. J Refract Surg. 2018;34(7):489-96.

52. Kamiya K, Shimizu K, Igarashi A, Kitazawa Y, Kojima T, Nakamura T, et al. Posterior chamber phakic intraocular lens implantation: comparative, multicentre study in 351 eyes with low-to-moderate or high myopia. $\mathrm{Br} \mathrm{J}$ Ophthalmol. 2018;102(2):177-81.

53. Lee J, Kim Y, Park S, Bae J, Lee S, Park Y, et al. Long-term clinical results of posterior chamber phakic intraocular lens implantation to correct myopia. Clin Exp Ophthalmol. 2016;44(6):481-7.

54. Kojima T, Kitazawa Y, Nakamura T, Takahashi M, Kamiya K, Ichikawa K, et al. Prospective randomized multicenter comparison of the clinical outcomes of $v 4 c$ and $v 5$ implantable collamer lenses: a contralateral eye study. J Ophthalmol. 2018;2018:7623829.

55. Yaşa D, Ürdem U, Ağca A, Yildirim Y, Kepez Yildiz B, Kandemir Beşek N, et al. Early results with a new posterior chamber phakic intraocular lens in patients with high myopia. J Ophthalmol. 2018;2018:1329874. 